\title{
Seven Life Lessons from Humanistic Behaviorism: How to bring the best out of yourself and others
}

\author{
E. Scott Geller \\ Virginia Tech
}

\begin{abstract}
Seven evidence-based guidelines for improving the quality and increasing the frequency of desirable behavior are described and illustrated as relevant for benefiting human welfare and well-being. If practiced extensively, these life lessons would most assuredly improve overall quality of life by reducing interpersonal conflict and bullying, preventing the occurrence of unintentional injuries and fatalities, and enhancing work productivity, environmental sustainability and life satisfaction. The first three guidelines reflect the applied behavioral science principles of positive reinforcement, observational learning, and behavior-based feedback. The subsequent four life lessons are essentially derived from humanism. Techniques for operationalizing these humanistic guidelines are presented, demonstrating social validity in integrating select principles from humanism with behaviorism. The result: humanistic behaviorism -- the application of some humanistic fundamentals to make behaviorism more acceptable, effective, and sustainable on a large scale.
\end{abstract}

Keywords: humanistic behaviorism, behavioral feedback, empathy, management vs. leadership, self-motivation, self-transcendence 


\title{
Seven Life Lessons from Humanistic Behaviorism: How to bring the best out of yourself and others ${ }^{1}$
}

\author{
E. Scott Geller \\ Virginia Tech
}

Suppose you were asked to define and explain the top seven lessons you've learned from studying the literature of behavioral and psychological science. Not just the most memorable, important, or most researched lessons, but those evidence-based lessons you believe should be taught and disseminated worldwide to benefit human welfare and quality of life. Which would you choose? Before reading further, it would be beneficial to ponder this question and derive your own list of seven life lessons. Then compare your list with those discussed here, noting similarities and discrepancies.

Obviously there's no right answer to the question: "What are seven crucial life lessons from psychological science?" Answers certainly will be biased by personal experience, including idiosyncratic reading of a diverse literature and varied research experiences at an educational and/or research institution and beyond. The seven life lessons presented here are derived from my selective perception, which evolved from intensive and extensive study of human dynamics - five years in graduate school and 45 years as a teacher and researcher of psychology at Virginia Tech.

The first three lessons connect directly to applied behavioral science (ABS), and will likely be included in the lists of most readers. The remaining four life lessons reflect humanism,

\footnotetext{
${ }^{1}$ This article was adapted from the author's keynote presentation at the Association for Behavior Analysis International Seminar on Leadership and Cultural Change, May $25^{\text {th }}, 2014$. The author is grateful for informational and inspirational feedback from the following individuals who read a prior draft of this article: Katya Davydova, Cory Furrow, Rob Holdsambeck, Georgiana Lee, Shane McCarty, Micah Roediger and two anonymous reviewers.
} 
and are likely not among the life-lesson lists created by readers of $J O B M$. However, an aim of this article is to convince you these life-lessons should be accepted, even deemed important, by students and teachers of ABS and organizational behavior management (OBM). Perhaps some readers will consider the life lessons derived from humanism to be an overly "radical" departure from behaviorism.

Recall that the "radical" adjective in "radical behaviorism" was used to denote deviance from the narrow "methodological behaviorism" advocated by Watsonian psychologists to infer the absence of unobservables. As explained by Ogden Lindsey (1991), this is "nothing-elsebehaviorism" for "those who say there is only observable behavior, nothing else." (p. 452). In contrast, "Skinner's new radical behaviorism not only accepted thoughts and feelings, but also put their causes in the environment along with outer behavior's causes" (p. 453). So how radical is humanistic behaviorism?

\section{Humanistic Behaviorism}

To be sure, this article is not the first to entertain the concept of humanistic behaviorism. More than 40 years ago, F. William Dinwiddie (1975) proposed humanistic behaviorism as "a working model for modern, dynamic, and successful treatment centers for children (because)... behaviorism modulated by traditional humanistic approaches helps in the molding of an efficient helping environment for children" (p.259). Similarly, Carl E. Thoresen (1972) claimed "... humanistic psychology offers directions for the kind of behavior that individuals should be able to engage in; contemporary behaviorism offers principles and procedures to help individuals increase their humanistic actions" (p. 4).

In fact, a number of behavioral scientists in the 1970s considered themselves humanists (e.g., Day, 1971; Hosford \& Zimmer, 1972; Kanfer \& Phillips, 1970; Lazarus, 1971; 
MacCorquodale, 1971; Staats, 1971; Thoresen \& Mahoney, 1974; Ullman \& Krasner, 1969) because they: a) focused on individual behavior under present circumstances; b) emphasized the role of learning in explaining and resolving human problems; c) examined how environments can be changed to prevent or alleviate human problems; and d) used the scientific method to develop and improve intervention techniques (Thoresen, 1972). Given these criteria, B.F. Skinner would be considered a humanist, and indeed he was honored with "Humanist of the Year" in 1972 by the American Humanists Association, founded in 1941 to be a clear and democratic voice for humanism in the U.S. and to develop and advance humanistic thought and action (American Humanistic Association, 2008).

Yes, integrating humanistic and behavioristic concepts was proposed by several scholars in the 1970s, but since then there has been very little discussion of this notion, especially for behavior change beyond the clinic. Few, if any, students in introductory psychology courses read or hear the term humanistic behaviorism. Instead, most introductory psychology textbooks focus on explicating distinct differences between the humanistic and behavioristic approaches to clinical therapy.

It's not difficult to find critics of integrating humanism and behaviorism. Newman (1992) censured an alliance between humanism and behaviorism, claiming, "The unfortunate truth is that many problems in living will not be alleviated by empathy, a supportive environment or even unconditional positive regard" (p. 47). Plus, after reviewing Newman's book, The Reluctant Alliance: Behaviorism and Humanism, Houts (1993) concluded, "Based on the evidence we have thus far, there is little reason to believe that integrating humanistic psychotherapy and education with behavior analysis will do anything but attenuate the efforts of behavior analysis" (p. 70). 
Please note that this critique of a humanistic behaviorism alliance occurred more than two decades ago within the context of clinical therapy. Plus, the integration of humanism and behaviorism proposed in this article selects certain (not all) concepts from humanism to augment the impact of behavior-focused intervention. These humanistic concepts are included as life lessons based on empirical evidence (e.g., perceptions that increase self-motivation and a sense of empowerment); others are not included for lack of research support (e.g., unconditional positive regard).

Bottom line: The notion of humanistic behaviorism is certainly not new, and integrating these seemingly disparate domains to develop sustainable behavior-change interventions for the large-scale benefit of individual and group health and well-being is not particularly innovative. Thus, the seven life lessons derived from humanistic behaviorism are not that radical from radical behaviorism.

\section{Employ the Power of Positive Consequences.}

Skinner's concern for people's feelings and attitudes is reflected in his antipathy toward the use of punitive consequences to motivate behavior. "The problem is to free men, not from control, but from certain kinds of control” (Skinner, 1971, p. 5). Skinner proceeded to explain that control by negative consequences must be reduced to increase perceptions of personal freedom.

The same situation can usually be viewed as: a) control by penalizing unwanted behavior, or b) control by rewarding desired behavior. Some students in my university class, for example, are motivated to avoid failure (e.g., a poor grade); while other students are motivated to achieve success (e.g., a good grade or increased knowledge). Which students feel more empowered and in control of their class grade? Which have a better attitude toward the class? Of course, you 
know the answer. Reflect on your own feelings or attitude in similar situations where you perceived your behavior was influenced by either positive or negative consequences.

Figure 1 depicts four distinct achievement typologies defined by Covington (1992), and derived from the seminal research of Atkinson $(1957,1964)$. These four classifications have been researched to explain differences in how people approach success and/or avoid failure (Covington \& Roberts, 1994). It's most desirable to be a success seeker. These are the optimists who respond to setbacks (e.g., to corrective feedback) in a positive and adaptive manner. They are self-confident and willing to take risks, as opposed to avoiding challenges to avoid failure. They wake up each day to an opportunity clock rather than an alarm clock. It's a mindset or attitude toward life you can influence in yourself and others with situational manipulations.

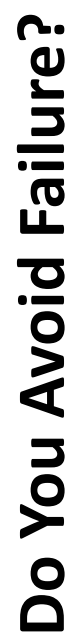

Do You Seek Success?

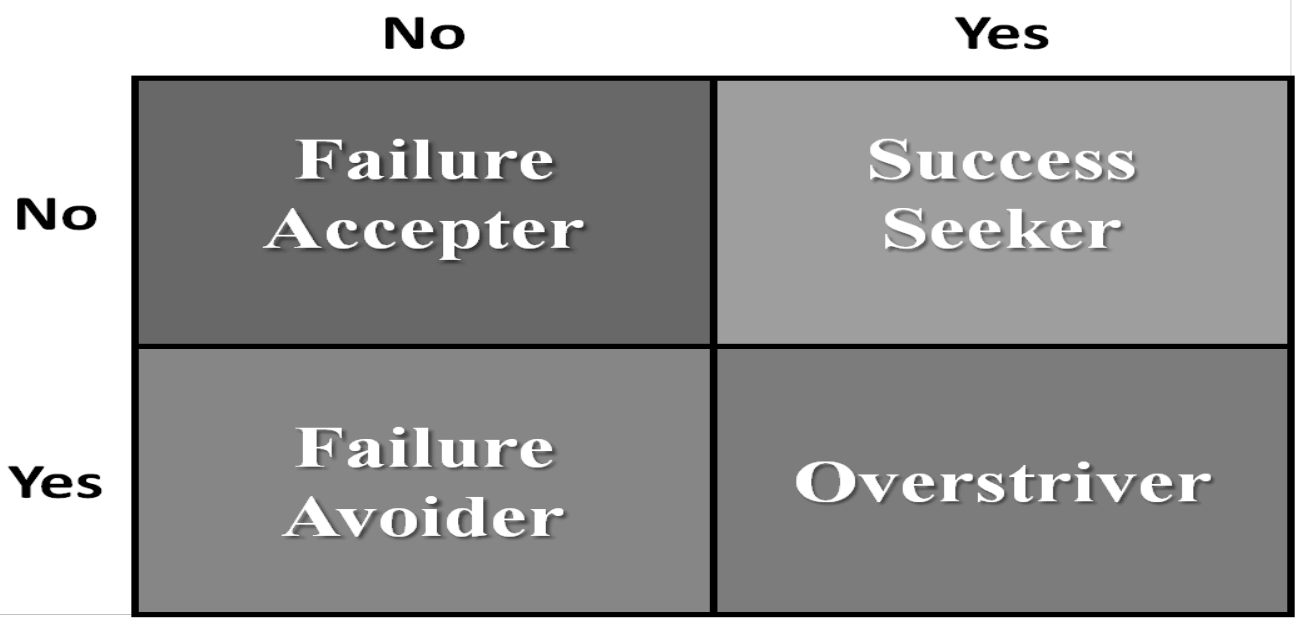

Figure 1. The four motivational typologies defined by achieving success vs. avoiding failure

Failure avoiders have a low expectancy for success and a high fear of failure. They do whatever it takes to protect themselves from appearing incompetent. They often use selfhandicapping and defensive pessimism to shield themselves from experiencing failure (Berglas \& Jones, 1978). These individuals are motivated but are not "happy campers". They are the 
students who say, "I've got to go to class; it's a requirement," rather than, "I get to go to class; it's an opportunity".

Bottom line: Applying soon, certain and positive consequences is the most efficient way to improve behavior and attitude at the same time. Of course, every reader of this article knows this, and also knows the term "positive reinforcer" should only be used if the positive consequence increases the frequency of the target behavior. But even when positive consequences do not result in an observable change in behavior, they are still useful. They might, in fact, affect an unobserved behavior, and they likely benefit an attitude or person-state, possibly enhancing a success-seeking mindset. These consequences are called rewards, as you know.

Using this life lesson on a daily basis is both critical and challenging. Why? Because we seem to live in a "click-it-or-ticket" culture that relies on negative reinforcement and punishment to manage behavior, from the classroom and workplace to our homes, and during our travel in between. The government approach to behavior management is to pass a law and enforce it. It's not enough to understand and believe this life lesson, we need to act accordingly. Thus, our next life lesson.

\section{Exercise Observational Learning.}

If you want to be better at what you do, watch someone who performs that behavior better than you. Of course, we all realize the power of observational learning. Indeed, a large body of psychological research indicates this type of learning is involved to some degree in almost everything we do (Bandura, 1969). Our actions influence others to a greater extent than we realize. Children learn by watching us at home and our colleagues are influenced by our 
actions at work. We're often unaware of such influence. Consider what children learn by watching the driving behavior of their parents, including their parent's verbal behavior.

It's likely observational learning is key to the dramatic success of behavior-based safety (BBS) at preventing injuries. Consider the basic BBS process: a) Coworkers develop a checklist of critical safe and at-risk behaviors on their job, termed a critical behavior checklist (CBC); b) They observe each other while working, and systematically check the safe and at-risk behaviors they observe; and c) The observer shows the completed CBC to the worker observed, and they discuss the results.

The role of observational learning is obvious. The observers note safe work practices, and might learn new ways to work safely in the process. They also observe at-risk behaviors to avoid, perhaps behaviors they themselves perform all too often. This representation of observational learning suggests BBS observers will subsequently work more safely on similar work tasks. Yes, this logical supposition from observation learning has been demonstrated empirically (e.g., Alvero \& Austin, 2004; Alvero, Rost, \& Austin, 2008). Of course, a most important component of the BBS peer-to-peer observation process is behavioral feedback -- our third life lesson.

\section{Give and Receive Behavioral Feedback.}

"Practice makes perfect" is not true; practice makes permanence. Only with behaviorbased feedback can practice improve behavior. Sometimes behavioral feedback is a natural consequence, as when the golfer and tennis player see where their ball lands after swinging a golf club or tennis racket. But even when we observe the outcome of our behavior, behavioral feedback from an observer (e.g., a coach) is necessary for proper behavioral adjustment and improvement. 
Most readers of this article would include this life lesson among their top seven. Indeed, most effective OBM interventions include some form of behavioral feedback, as does BBS. The letters of COACH say it all: “C” for Care; “O” for Observe; “A” for Analyze, “C” for Communicate, and "H” for Help (Geller, 2001).

It all starts with Caring. 'Know I Care and you'll care what I know. I care so much, I'm willing to Observe you and record occurrences of desirable and undesirable behavior." The observer records occasions of designated behaviors, and notes environmental factors that may be influencing the observed behavior, from antecedent conditions to behavioral consequences. This is the Analysis phase of coaching.

Next, we have interpersonal Communication - the delivery of information gained from the prior Observation and Analysis steps. Most people want to improve; but many people resist giving and receiving the kind of communication that is critical to beneficial behavior change. Some people perceive feedback that implies personal change as an indictment of their current work style, job skills, or diligence. This reaction is most likely to happen when someone is being asked to change dramatically, and when current procedures have been followed for years. To overcome this resistance, effective behavior-improvement coaches steer clear of disruptive and dramatic communication and emphasize incremental fine tuning or successive approximations. They also facilitate beneficial change to both behavior and attitude by accentuating the positives - occurrences of desirable behavior.

Like client-centered or humanistic therapy (Rogers, 1942), the focus is on the perceptions and feelings of the individual being coached. Behavioral and environmental conditions are observed from this person's perspective, and feedback communication is supportive and 
nondirective. Feedback is typically not delivered to direct behavior change, but to empower personal acceptance and self-accountability for designated behavioral improvement.

If the interpersonal communication goes well, the last letter of $\mathrm{COACH}$-- Help -- is accomplished. The behavioral feedback was accepted and will be used to improve the pinpointed behaviors. Note how the four letters of HELP -- Humor, Esteem, Listen, Praise -reflect strategies to increase the probability a coach's advice, directions, or feedback will be appreciated.

\section{Replace the Golden Rule with the Platinum Rule.}

We've all been exposed to the Golden Rule: "Treat others the way you want to be treated." Let's consider the Platinum Rule (Allesandra \& O'Connor, 1996): "Treat others the way they want to be treated." This viewpoint reflects the idiographic philosophy of both humanism and behaviorism. The uniqueness of each person is considered, and generalization across people and their experiences is avoided. It's psychology as the study of the individual -- not the group. Indeed, Skinner's experimental study of single rats and pigeons in a within-subject ABA reversal design implies an idiographic approach to behavioral science (Skinner, 1938, 1953).

Employing the Platinum Rule positions us to better serve and meet the specific needs of others. This is essential to establishing a brother/sister keepers' culture of people looking out for the welfare of each other. It enables the development of authentic interpersonal relationships and the community spirit needed for behavioral feedback to be given and received frequently, constructively, and in a non-adversarial manner. When these relationships develop in the workplace, the ability to inspire and influence the achievement of all business objectives is optimized. 


\section{Embrace and Practice Empathy.}

The rationale for using more positive than negative consequences to motivate behavior is the differential feeling states provoked by positive versus negative reinforcement, and punishment. Similarly, the way an intervention process is implemented can increase or decrease feelings of empowerment, build or destroy trust, and cultivate or inhibit a sense of teamwork or belonging (Geller, 2005). It's important to assess person-states or perceptions occurring concomitantly with an intervention process. This can be accomplished informally through oneon-one interviews and group discussions, or formally with a perception survey (O'Brien, 2000; Peterson, 2001).

Decisions regarding which $\mathrm{ABS}$ intervention to implement, and how to refine existing intervention procedures should be based on both objective observations of behaviors and subjective evaluations of feeling states. Often, it's possible to employ empathy to evaluate the indirect internal impact of an intervention. Imagine yourself going through a particular set of intervention procedures. Then, ask yourself, "How would I feel?"

\section{A Personal Example}

Two decades ago when my daughter wanted to drive my car to her high school I installed a large sign on the back, with the bold message, "How's my driving? Call my Dad: 540-6267712." I bolted the sign to my vehicle after she had received a percent-safe score of $100 \%$ on three consecutive coaching sessions with our $\mathrm{CBC}$, as described under Life Lesson 2. We had this "if-then" contingency: "Achieve a perfect score on three consecutive trips with the $\mathrm{CBC}$, and you may drive my car to school."

I was sure she'd accept the addition of the sign on my vehicle. Note how this activator is more than an awareness prompt; it implies a consequence. We had talked frequently about the 
value of positive or supportive consequences, so I thought Krista would view this sign as a "fun" and positive approach to promote safe driving. "Let's be optimistic about this," I said to her, "and see how many positive phone calls I get about your safe and courteous driving behavior."

"Are you kidding me, Dad, there's no way I'd park that car and sign at my high school," Krista retorted. "I'd be the laughing stock of the whole school. I'll talk to Mom about this." ${ }^{2}$ My lesson: Don't assume you know how a well-intentioned intervention will be received by the participant(s); ask first.

Empathic listening, diagnosing, and action planning take patience. Conversations at this level are often not efficient, but they are always most effective. Through questioning and listening, the objective is to first learn what it's like to be in the other person's situation. Then the objective shifts to developing a corrective intervention that fits the circumstances as mutually understood by everyone involved in the conversation. If commitment to follow through with a specific action plan is stated, you were an empathic behavioral coach.

\section{A Critical Caveat}

Before proceeding to the next life lesson, consider a crucial exception to an intervention approach implied by these latter humanism-derived life lessons -- practicing empathy to follow the Platinum Rule. Can you think of circumstances where treating others the way they want to be treated could do more harm than good? Are there times when adherence to the Platinum Rule is detrimental to human welfare?

Parents, teachers, and readers who have used ABS to improve the behavior of children can list numerous situations where the behavioral target of an intervention had to be defined by the parent, teacher, coach, or behavior analyst; not by the child. Simply put, children don't

\footnotetext{
${ }^{2}$ Subsequently, Krista earned a Ph.D. in Human Development and teaches each of these life lessons as the Global People-Based Safety and Human Performance-Improvement Manager for Bechtel Corporation.
} 
always know what's good for them; and when they do (e.g., complete house chores or a homework assignment), they might not choose that behavior to be the focus of a behaviorchange intervention.

How about BBS? Treating workers the way they want to be treated implies they should be allowed to take risky shortcuts and avoid wearing uncomfortable personal protection equipment. And, if we listened with empathy to the public, vehicles would not even have safety belts, let alone laws requiring their use; and drivers could travel at any speed they deem safe, while engaging in all sorts of distracting behaviors. Plus, cigarette smokers could practice their unhealthy behavior where ever they wanted.

Obviously, the Platinum Rule can be stretched to absurdity. There are numerous occasions when people of all ages should not be treated the way they want to be treated. Behaviors defined for the public "good" often conflict with egoistic wishes; and in these cases, the Platinum Rule can't work. But the traditional Golden Rule is also irrelevant if the person doing the "treating" is also egotistically disregarding a policy or rule (e.g., a safety or health regulation), or performing a self-serving but public-destructive behavior (e.g., smoking a cigarette in a public setting, or driving a vehicle while intoxicated).

To influence people to sacrifice personal pleasures detrimental to public welfare, interventions must be implemented to increase the frequency of behaviors that benefit large-scale well-being. But these behaviors might not be intrinsically reinforcing to the individual. Here's where the first three life lessons are paramount -- positive consequences, modeling, and behavioral feedback. In this regard, please note a critical incongruity between behaviorism and humanism. 
The first life lesson employs positive consequences conditional on the occurrence of a desirable behavior; whereas the humanistic therapy of Carl Rogers (1942) features unconditional positive regard. Although a love-them-anyway approach can work at times, readers of this article realize the behavior-change advantages of conditional positive regard. Thus, the life lessons explicated here implicate some but certainly not all tenets of humanism.

\section{Distinguish between Managing Behavior and Leading People.}

Management is not the same as leadership. Yes, both are critically important for bringing the best out of people. Simply put, managers hold us accountable to perform desirable behavior and avoid undesirable behavior. Leaders inspire us to hold ourselves accountable to do the right thing. Managers control behavior with an external (or extrinsic) accountability intervention or system. Leaders facilitate self-motivation by influencing person-states (e.g., perceptions, attitudes, and/or emotions) that facilitate self-motivation. Self-motivation (or self-directed behavior) often leads to discretionary behavior (Daniels \& Daniels, 2005) -- behavior beyond that which is required.

\section{Self-Motivation}

The C-words of Choice, Competence, and Community are used by Geller and Veazie (2010) in their narrative to illustrate the three evidence-based perceptions or person-states that determine self-motivation. Dispositional, interpersonal, and environmental conditions that enhance these states, presumed to be innate needs by some psychologists (Deci, 1975; Deci \& Flaste, 1995; Deci \& Ryan, 1995), increase personal perceptions of self-motivation. Guidelines for enhancing perceptions of choice, competence, and community are given elsewhere (Geller, 2014a,b), and many of these are consistent with the life lessons discussed here. Consider, for example, how proper application of Lessons 1,2,3, and 5 can increase one's perception of 
competence and hence fuel self-motivation. Consider, also, how our language can affect each of these perceptions.

Watch your language. Your language should suggest minimal external pressure. The common phrases: "Safety is a condition of employment," "All accidents are preventable," and "Bullying is a rite of passage," reduce one's sense of autonomy. In contrast, the slogan, "Actively caring is a core value of our organization" implies personal authenticity, interpersonal relatedness, and human interaction.

The common phrase "random acts of kindness" (Editors of Conari Press, 1993) has a disadvantage when describing or promoting prosocial behavior. Random implies the behavior happens by chance, suggesting it's beyond individual choice or control. The kind act may appear random to the recipient, but it was likely performed intentionally and was self-motivated. An alternative: "intentional acts of kindness." The language we use to prescribe or describe behavior influences our perceptions of its meaningfulness and its relevance to our lives. Language impacts culture, and vice versa.

Opportunities for choice. Participative management means employees have personal choice during the planning, execution, and evaluation of their jobs. People have a need for autonomy, regardless of dispositional and situational factors. In the workplace, managers often tell people what to do rather than involving them in the decision-making process. Referring to language again, should managers give "mandates" or set "expectations"? Should they request "compliance" or ask for "commitment"?

In schools, students are often viewed as passive learners, because teachers plan, execute, and evaluate most aspects of the teaching/learning process. Students' perceptions of choice are 
limited. Yet cooperative teaching/learning - where students contribute to the selection and presentation of lesson material - is most beneficial over the long term (Chance, 2008).

Involving the followers. Autonomy is supported when rules are established by soliciting input from those affected by the regulation (Deci \& Flaste, 1995). Employees are more likely to comply with safety regulations they helped to define. Shouldn't they have substantial influence in the development of policy they will be asked to follow? Those on the "front line" know best what actions should be avoided versus performed in order to optimize the safety and quality of their production system.

Similarly, before a rule or regulation is implemented in an educational system, those affected (i.e., faculty and/or students) should certainly be given opportunities to offer suggestions. In a family, as the children mature, certain rules should be open to discussion before being imposed. This takes more time, but the marked increase in effectiveness justifies the loss in efficiency.

\section{Empowerment}

In the management literature, empowerment typically refers to delegating authority or responsibility, or to sharing decision-making (Conger \& Kanungo, 1988). In other words, when a manager says, "I empower you," s/he usually means, "Get 'er done." In contrast, the empathic leader first assesses whether the "empowered" individual feels empowered. "Can you handle the additional assignment?" Proper assessment of feeling empowered involves asking three questions, as derived from social learning theory (Bandura, 1997).

As depicted in Figure 2, the first question, "Can I do it?" is asking whether the empowered individual or group has the resources, time, knowledge, and ability to handle the assignment. The knowledge and ability components refer to training, and the term self-efficacy 
places the focus on personal belief. An observer might think an individual has the competence to complete a task, but the empowered person might feel differently. Thus, a "yes" answer implies a belief of relevant personal effectiveness by those who received the assignment or who set a performance-improvement process goal.

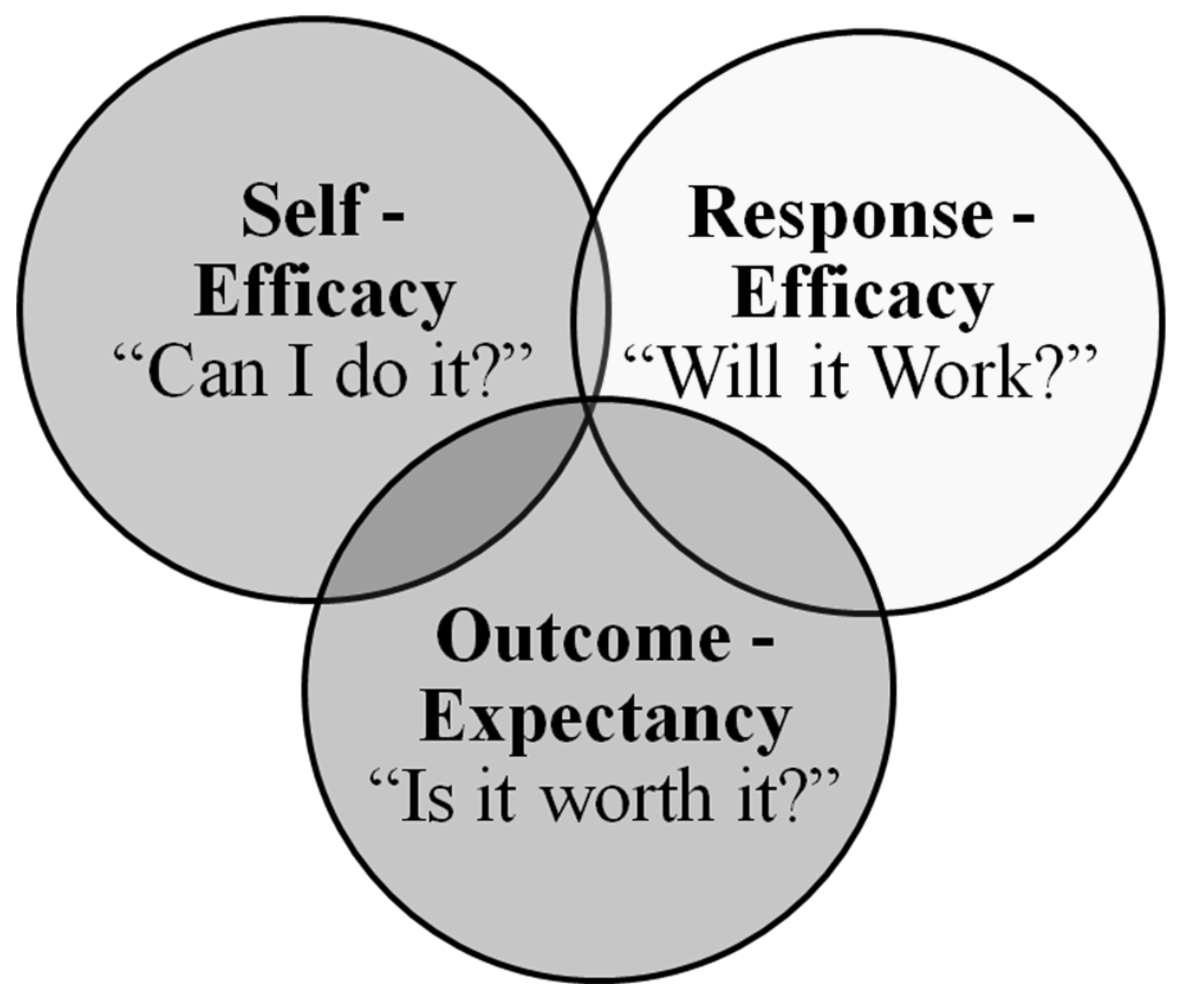

Figure 2. The three beliefs that determine empowerment (adapted from Bandura, 1997)

The second question, the response-efficacy question, asks whether those empowered believe pursuing and accomplishing the assignment or attaining the process goal (i.e., performing the required behaviors) will contribute to a valued mission of the organization, work team, or individual. Regarding workplace safety, this translates to believing a particular injuryprevention process (e.g., a BBS observation-and-feedback process) will contribute to achieving the vision of an injury-free workplace.

A sports team would answer "yes" to this question if the members believe their new workout routine or competition strategy will increase the probability of winning a game. And, 
the student studying for an exam would give a "yes" answer to response-efficacy if s/he believes the study strategy will contribute to earning a higher exam grade. Of course, the behavioral outcome for these two examples could be more distal and substantive, like having a winning season or obtaining a college degree, respectively.

Whereas a "yes" answer to the "self-efficacy" question might require more training, education might be needed to obtain a "yes" answer to the response-efficacy question. In other words, people might believe they can accomplish a particular process or task, but not believe such accomplishment will make a difference in an ultimate outcome. In this case, education is needed, including an explanation of an evidence-based principle or theory and perhaps the presentation of convincing data.

The third empowerment-assessment question targets motivation. Is the expected outcome worth the effort? The performance of relevant behavior is motivated by anticipating a positive consequence to achieve or a negative consequence to avoid. Referring back to the first Life Lesson, recall that people feel more choice and are more likely to be self-motivated when they are working to achieve a positive consequence than when they are responding to avoid a negative consequence.

Empowering goals. Readers of this journal are well aware of the beneficial role of behavior-focused goal setting as an activator of process activities aimed at achieving a particular outcome. They are also likely aware of a popular acronym used to define the characteristics of an effective goal: SMART. There are actually a few variations of the words reflected by these acronym letters, with $M$ representing Measurable or Motivational and $T$ referring to Timely or Trackable, for example. 
I proposed the following acronym words: $S$ for Specific, $M$ for Motivational, $A$ for Attainable, $R$ for Relevant, and $T$ for Trackable (Geller, 2005, 2008) -- later adding $S$ (i.e., SMARTS) for Shared, because special support can increase commitment to work toward reaching a goal (Geller, 2014c). A rationale and procedural details for each component of effective goal setting are provided elsewhere (Geller, 2005, 2014c). Here it's instructive to note the connection between SMARTS goals and the empowerment model introduce earlier.

Specifically, SMARTS goals are empowering because they are attainable ("I can do it."), motivational ("It's worth it."), and relevant ("It will work."). This connection makes it clear that both empowerment and goal setting are similar behavioral antecedents, setting the stage (or activating) certain behavior(s). Each of these establishing operations refers to motivation as the anticipation of a desirable consequence or outcome.

Empowerment vs. self-motivation. It's important to consider a critical distinction between these two person-states. Empowerment is a behavioral antecedent or establishing operation (Michael, 1982), whereas self-motivation reflects the impact of consequences. In other words, feeling empowered means the individual is ready (or activated) to work toward achieving a given goal. On the other hand, a self-motivated person is anticipating or has received a consequence that supports self-directed rather than other-directed behavior (Watson \& Tharp, 1997).

Although the initial proponents of self-motivation (Deci, 1975, Deci \& Ryan, 1995) did not connect self-motivation with behavioral consequences, a behavioral scientist naturally associates consequences with motivation, as implied by Skinner's legacy, "selection by consequences" (Skinner, 1981). With this perspective, consequences that reflect personal choice, competence, and/or a sense of social support or community should enhance self-motivation and 
thereby increase the durability of a behavior-change intervention that employs positive reinforcement. Thus, an intervention applying positive consequences to increase the occurrence of a target behavior should have longer-term impact if the intervention inspires self-motivation by linking the behavioral consequence(s) with a perception of choice, competence, and/or community. Perhaps some readers will view this as a hypothesis worthy of ABS research.

\section{Progress from Self-Actualization to Self-Transcendence.}

This final life lesson connects most obviously to humanism, but without ABS it's just a theory with limited practical value. Indeed, a transition to self-transcendence could be key to saving the world from itself (Skinner, 1971).

\section{A Hierarchy of Needs}

Probably the most popular theory of human motivation is the hierarchy of needs proposed by humanist Abraham Maslow (Maslow, 1943). Categories of needs are arranged hierarchically. It's presumed people don't attempt to satisfy needs at one stage or level until the needs at the lower stages are satisfied.

First, we are motivated to fulfill physiological needs. This includes food, water, shelter, and sleep for basic survival. After these needs are met, we are motivated by the desire to feel secure and safe from future dangers. When we prepare for future physiological needs, we are working proactively to satisfy our need for safety and security.

Next, we have our social-acceptance needs--the need to have friends and feel like we belong. When these needs are gratified, our concern shifts to self-esteem, the desire to develop self-respect, gain the approval of others, and achieve personal success. Now we have "selfactualization," right? Is this the highest level of Maslow's Hierarchy of Needs? No, it's not. 
Maslow's Hierarchy of Needs is illustrated in Figure 3. Note self-actualization is not at the top. Maslow revised his renowned hierarchy shortly before his death in 1970, placing selftranscendence above self-actualization (Maslow, 1971). Transcending the self means going beyond self-interest to actively care for others.

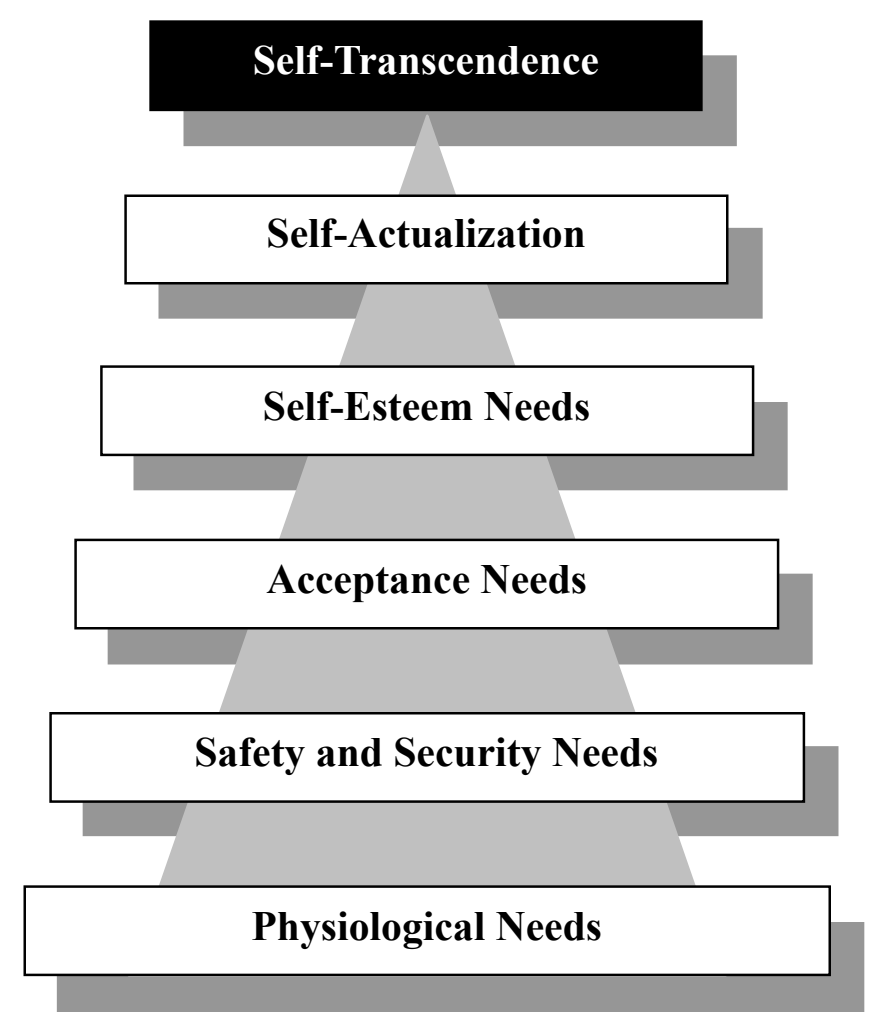

Figure 3: Maslow's revised hierarchy which shows self-transcendence at the top

It seems intuitive that various self-needs require satisfaction before self-transcendent or actively-caring-for-people (AC4P) behavior is likely to occur. But scant research supports the ranking of human needs in a hierarchy. It's possible to think of many examples where individuals performed various AC4P behaviors before satisfying all of their personal needs. Mahatma Gandhi put the concerns of others before his own. He suffered imprisonment, 
extensive fasts, and eventually assassination in his 50-year struggle to help his poor and downtrodden compatriots.

Note the connection between the need hierarchy and various potentially-reinforcing consequences, as well as our discussion of self-motivation and sustaining the impact of a behavior-change intervention. An individual's position in the hierarchy certainly determines what types of consequences are likely to be most reinforcing at a particular time. Without food, shelter or sleep, for example, most people will focus their efforts on satisfying these needs. But if this need level is satisfied (i.e., an establishing operation of satiation), the motivation of human behavior requires consequences related to higher-level needs.

As we climb Maslow's hierarchy, need states are reached that implicate consequences linked to self-motivation. For example, consequences that boost one's sense of connection with others (i.e., community) satisfy the need for acceptance or social support, and consequences that certify a person's belief in personal competence to perform worthwhile work are associated with the self-esteem and self-actualization needs. Plus, it's intuitive that reaching beyond self-needs to help others through AC4P behavior can contribute to satisfying a person's need for social acceptance and self-esteem, and even self-actualization.

Question: When does one's need for social acceptance, self-esteem, and self-actualization get satisfied? In other words, at what point does a person become satiated on consequences linked to these need states? Yes, these are rhetorical questions, posed to reiterate the value of delivering rewarding consequences that reflect the three C-words of self-motivation (i.e., Competence, Choice, and Community) and enhances need states in Maslow's hierarchy that are difficult to satiate. In other words, behavioral consequences that foster perceptions of personal competence, self-worth, interpersonal belongingness, and/or autonomy also facilitate self- 
motivation and self-directed behavior, and are thus likely to have longer-term impact than consequences unrelated to these person-states.

\section{The AC4P Movement}

I coined the term "actively caring" in 1990 when working with a team of safety leaders at the Exxon Chemical facility in Baytown, Texas (Geller, 1991). The vision was to cultivate a brother/sister keepers' culture in which everyone looks out for each other's safety -- people routinely going above and beyond the call of duty to benefit the health, safety, and/or welfare of others.

"Actively caring for people" was an ideal description for this site-wide paradigm shift. Most people care about the well-being of others, but relatively few "act" on their feelings of caring. The challenge was to get everyone to actively care-- to take effective action based on their caring. The vision: a company with more interpersonal empathy, compassion, and AC4P behavior.

Following the Virginia Tech campus-shooting rampage on April 16, 2007 which took the lives of 33 students and faculty and injured 17 others (Geller, 2008b), the mission of AC4P took on a new focus and prominence for me and my students. In a time of great uncertainty and reflection, those most affected by the tragedy were not thinking of themselves. They acted to help classmates, friends, and even strangers. This collective effort was manifested in an AC4P Movement for worldwide culture change.

For more than 20 years, I promoted the use of a green wristband embossed with the words "Actively Caring for People" to recognize individuals for their AC4P behavior. During those years, I distributed about 50,000 of these wristbands following his keynote addresses at conferences and organizations. Recently, the author's students have used this recognition 
approach to reduce bullying by promoting and rewarding AC4P behavior in various educational settings (McCarty \& Geller 2011; McCarty, Teie, McCutchen, \& Geller, in press).

For these latter applications, the AC4P wristbands were redesigned to include a different identification number per wristband, and the website (www.ac4p.org) was developed for people to: a) share their AC4P stories (with the number of the wristband they gave or received), b) track worldwide where a particular AC4P wristband has been, and c) order more AC4P wristbands to reward others for their AC4P behavior.

It's recommended the delivery of an AC4P wristband should be accompanied with words that serve higher-level needs. For example, never suggest the wristband is a "pay-off" for AC4P behavior; rather, the wristband is only a token of appreciation for the "special servant leadership exemplified by the observed act of kindness." Plus, the wristband recipient is told s/he is now one of many who have joined the AC4P Movement -- a flourishing worldwide effort to cultivate cultures of interpersonal compassion and interdependent AC4P behavior.

To date, more than 4,000 AC4P stories have been shared at this website, and more than 100,000 AC4P wristbands have been purchased, with proceeds going to the Actively Caring for People Foundation, Inc. It's hoped this particular accountability system for activating and rewarding AC4P behavior will help to spread the AC4P paradigm worldwide, and inspire the development of AC4P cultures in various settings.

\section{In Summary}

This article introduced seven life lessons derived from behaviorism and humanism, and presumed to benefit human well-being and quality of life wherever they are practiced. The first three lessons are employed in almost every successful intervention developed and evaluated by OBM researchers and practitioners. The next four connect directly to the principles of 
humanism, and it's likely most readers of this journal will consider these to be beyond the empirical, behavior-based domain of OBM.

However, operational definitions were included with each of these humanistic lessons, making it possible to bring them to life with a behavior-focused intervention. For example, the concept of self-transcendence was discussed in terms of interpersonal AC4P behavior, and a practical application of Life Lesson 1 (i.e., the AC4P wristband) was illustrated to increase the frequency of AC4P behavior en route to achieving an AC4P culture.

Although a number of effective AC4P applications of the life lessons reviewed here have successfully improved human welfare (cf. Geller, 2014a,b), we've merely scratched the surface of societal problems that could be solved in part by large-scale applications of these life lessons. We have so much more to learn from the synergistic integration of applied behavioral science and humanistic psychology -- humanistic behaviorism.

\section{References}

Allesandra, T., \& O'Connor, M.S. (1996). The platinum rule: Discover the four basic business personalities and how they can lead you to success. NY: Warren Books, Inc.

Alvero, A. M., \& Austin, J. (2004). The observer effect: The effects of conducting behavioral observations on the behavior of the observer. Journal of Applied Behavior Analysis, 37, 457-468.

Alvero, A. M., Rost, K., \& Austin, J. (2008). The safety observe effect. Journal of Safety Research, 39(4), 365-373.

American Humanist Association. (2008). American Humanist Association. Retrieved June 16, 2014 from http://www.americanhumanist.org/ 
Atkinson, J. W. (1957). Motivational determinants of risk-taking behavior. Psychological Review, 64, 359-372.

Atkinson, J. W. (1964). An introduction to motivation. Princeton, NJ: Van Nostrand.

Bandura, A. (1969). Principles of behavior modification. NY: Holt, Rinehart \& Winston.

Bandura, A. (1997). Self-efficacy: The exercise of control. NY: W. H. Freeman and Company.

Berglas, S., \& Jones, E. E. (1978). Drug choice as a self-handicapping strategy in response to noncontingent success. Journal of Personality and Social Psychology, 36, 405-417.

Chance, P. (2008).The teacher's craft: The 10 essential skills of effective teaching. Long Grove, IL: Waveland Press, Inc.

Conger, J. A., \& Kanungo, R. N. (1988). The empowerment process: Integrating theory and practice. Academy of management review, 13(3), 471-482.

Covington, M. V. (1992). Making the grade: A self-worth perspective on motivation and school reform. NY: Cambridge University Press.

Covington, M. V., \& Roberts, B. W. (1994). Self-worth and college achievement: Motivational and personality correlates. In P. R. Pintrich, D. R. Brown, \& C. E. Weinstein (Eds.). Student motivation, cognition, and learning: Essays in honor of Wilbert J. McKeachie (pp. 157-187). Hillsdale, NJ: Earlbaum.

Daniels, A. C., \& Daniels, J. E. (2005). Measure of a leader. Atlanta, GA: Performance Management Publications.

Day, W.F. (1971). Humanistic psychology and contemporary humanism. The Humanist, 31, p. 13-16.

Deci, E. L. (1975). Intrinsic motivation. New York: Plenum 
Deci, E. L., \& Flaste, R. (1995). Why we do what we do: Understanding self-motivation. London: Penguin books.

Deci, E. L., \& Ryan, R. M. (1995). Intrinsic motivation and self-determinism in human behavior. NY: Plenum

Dinwiddie, F.W. (1975). Humanistic behaviorism: A model for rapprochement in residential treatment milieus. Child Psychiatry and Human Development, 5(4), p. 254-259.

Editors of Conari Press. (1993). Random acts of kindness. Emeryville, CA: Conari Press.

Geller, E. S. (1991). If only more would actively care. Journal of Applied Behavior Analysis, $24,763-764$.

Geller, E. S. (2001). The psychology of safety handbook. Boca Raton, FL: CRC Press.

Geller, E.S. (2005). People-based safety: The source. Virginia Beach, VA: Coastal Training Technologies Corporation.

Geller, E. S. (2008a). Leading people-based safety: Enriching your culture. Virginia Beach, VA: Coastal Training Technologies Corporation.

Geller, E. S. (2008b). The tragic shooting at Virginia Tech: Personal perspectives, prospects, and preventive potentials. Traumatology, 14(1), 8-20.

Geller, E. S. (2014a) (Ed.). Actively caring at your school: How to make it happen. Newport, VA: Make-A-Difference, LLC.

Geller, E. S. (2014b) (Ed.). Actively caring for people: Cultivating a culture of compassion. Newport, VA: Make-A-Difference, LLC.

Geller, E.S. (2014c). The psychology of self-motivation. In E.S. Geller (Ed), Actively caring for people: Cultivating a culture of compassion (pp. 73-75). Newport, VA: Make-ADifference, LLC. 
Geller, E. S., \& Veazie, R. A. (2010). When no one's watching: Living and leading selfmotivation. Virginia Beach, VA: Coastal Training and Technologies Corporation.

Hosford, R.E., \& Zimmer, J. (1972). Humanism through behaviorism. Counseling and Values, 16, p. 1-7.

Houts, A.C. (1993). Review of "The reluctant alliance: Behaviorism and humanism." Child \& Family Behavior Therapy, 15(4), p. 69-85.

Kanfer, F.H., \& Phillips, J.S. (1970). Learning foundations of behavior therapy. NY: Wiley, 1970.

Lazarus, A. (1971). Behavior therapy and beyond. NY: McGraw-Hill.

Lindsey, O.R. (1991). From technical jargon to plain English for application. Journal of Applied Behavior Analysis, 24, 449-458.

MacCorquodale, K. (1971). Behaviorism is a humanism. The Humanist, 31, p. 12-13.

Maslow, A. H. (1943). A theory of human motivation. Psychological Review, 50, 370-396;

Maslow, A. H. (1971). The farther reaches of human nature. NY: Viking.

McCarty, S. M., \& Geller, E.S. (2011). Want to get rid of bullying? Then reward behavior that is incompatible with it. Behavior Analysis Digest International. 23(2). pp 5, 7.

McCarty, S., Teie, S., McCutchen J., \& Geller, E. S. (in press). Actively caring to prevent bullying in an elementary school: Prompting and rewarding prosocial behavior. Journal of Prevention and Intervention in the Community.

Michael, J. (1982). Distinguishing between discrimination and motivational functions of stimuli. Journal of Experimental Analysis of Behavior, 37, 149-155.

Newman, B. (1992). The reluctant alliance: Behaviorism and humanism. Buffalo, NY: Prometheus Books, p. 47. 
O'Brien, D. P. (2000). Business measurements for safety performance. NY: Lewis Publishers.

Peterson, D. (2001). Authentic involvement. Itasca: National Safety Council.

Rogers, C. (1942). Counseling and psychotherapy. NY: Houghton Mifflin.

Skinner, B. F. (1938). The behavior of organisms: An experimental analysis. Acton, MA: Copley Publishing Group.

Skinner, B. F. (1953). Science and human behavior. NY: Macmillan.

Skinner, B. F. (1971). Beyond freedom and dignity. NY: Alfred A. Knopf

Skinner, B.F. (1981). Selection by consequences. Science, 213, 502-504.

Staats, A.W. (1971). Child learning, intelligence and personality. NY: Harper \& Row.

Thoresen, C.E. (1972). Behavioral humanism. Research and Development Memorandum No. 88, April, Stanford, CA: School of Education, Stanford University.

Thoresen, C.E., \& Mahoney, M.J. (1974). Behavioral self-control. NY: Holt, Rinehart \& Winston.

Ullmann, L.P., \& Krasner, L. (1969). A psychological approach to abnormal behavior. Englewood Cliffs, NJ: Prentice-Hall.

Watson, D.C., \& Tharp, R.G. (1997). Self-directed behavior: Self-motivation for personal adjustment (7th Edition). Pacific Grove, CA: Brooks/Cole Publishing. 